\title{
Desarrollo y democracia en América Latina: resultados y perspectivas
}

\section{Ronaldo Munck*}

Resumen. Tras una década y media de posneoliberalismo en América Latina, resulta conveniente examinar los desafíos del desarrollo democrático y las estrategias implementadas para satisfacerlos. Desde el año 2000 la transformación social en la región se debe a la aparición y consolidación de gobiernos de izquierda y movimientos sociales. Sin embargo, esta dinámica sociopolítica y su halo teórico no han tenido el impacto global que detentó la teoría latinoamericana de la dependencia en los 1970, debido a la diversidad y a la contradicción de las estrategias de gobiernos progresistas y movimientos sociales radicales. En ese sentido, el texto revisa los grandes problemas inherentes al desarrollo democrático: crecimiento, equidad, sustentabilidad y gobernabilidad. Además se exponen las políticas aplicadas: neoextractivismo, neodesarrollismo, socialismo del siglo XXI y Buen Vivir. Por último, realiza un análisis prospectivo acerca de los escenarios futuros del desarrollo democrático en América Latina.

Palabras clave: desarrollo, democracia, transformación social, análisis prospectivo, América Latina.

\footnotetext{
* Jefe de la oficina de Participación Cívica en la Universidad Ciudad de Dublín e investigador principal en el Instituto Ernesto Laclau de Estudios Interdisciplinarios de América Latina en la Universidad de Buenos Aires.

Traducción del inglés por Mauricio Alan Cabral Pérez.
} 


\section{Development and democracy in Latin America:} results and prospects

Abstract. Following a decade-and-a-half of post-neoliberalism in Latin Ameri$\mathrm{ca}$, it is appropriate now to examine the challenges of democratic development and the strategies implemented to address them. Since 2000, the social transformation in the region has been due to the appearance and establishment of leftist governments and social movements. However, this socio-political dynamic and its theoretical «halo» have not had the global impact expected from Latin American dependency theory of the 1970s, due to the diversity and the contradictions in strategies of progressive governments and radical social movements. In this sense, the article reviews the broad problems inherent in democratic development: growth, equity, sustainability and governability. It also outlines the policies that were applied: neo-extractivism, neo-developmentalism, socialism of the 21st Century and Buen Vivir. Lastly, it offers a forward-looking analysis of future scenarios in democratic develompent in Latin America.

Keywords: development, democracy, social transformation, prospective analysis, Latin America. 


\section{Desafíos}

Al examinar los principales desafíos para el desarrollo y la democracia desde una perspectiva latinoamericana, es posible proponer varios temas clave surgidos tras la larga noche de neoliberalismo: crecimiento, equidad, sustentabilidad y gobernabilidad. Esta es ahora una agenda ampliamente compartida, incluso para los otrora partidarios del neoliberalismo y tales desafíos incumben igualmente a gobiernos que favorecen al mercado o a la sociedad; es decir, en términos de dónde se encuentran los gobiernos con relación al doble movimiento planteado por Michael Polanyi: promover una lógica del mercado contra la sociedad o promover contramovimientos sociales que dominen las desnudas fuerzas del mercado (véase Munck, 2015). Al respecto se delinean dichos desafíos con el propósito de realizar más tarde un balance sobre la medida en que las fuerzas que buscan una alternativa al statu quo han subido a la palestra.

\section{Crecimiento}

Existe poco desacuerdo en el ámbito político - a excepción de algunos ambientalistas - de que el crecimiento económico sigue siendo el objetivo primordial para América Latina, al igual que en etapas anteriores. El periodo de posguerra en la región se caracterizó por un modelo de desarrollo hacia adentro, dirigido por el Estado, dedicado al consumo masivo. Alrededor de 1980 ocurre un giro brusco que conlleva a un modelo sustentado en la exportación, orientado por el mercado, donde el consumo masivo dejaba de ser esencial. En esa década el producto interno bruto (PIB) per cápita disminuyó en 0.8 por ciento, a consecuencia de una crisis 
de deuda masiva. Después, en la década de 1990 aumentó 1.5 por ciento en general, seguido por un significativo incremento de 2.1 por ciento en la década de 2000 (Bulmer, 2014:426). Sin embargo, esas tasas de crecimiento contrastan pobremente con otras regiones «en desarrollo» de Asia oriental. Desde 1980 Chile ha sido el único país que ha podido superar los índices de crecimiento de desarrollo hacia adentro del periodo 1950-1980. En 2015, en el contexto de una economía global lenta, América Latina creció 2.2 por ciento en promedio, lo que es razonablemente positivo.

La forma en que América Latina respondió a la crisis económica mundial de 2008-2009 ofrece un indicio de los avances logrados desde el punto más alto de la era neoliberal, cuando las fortunas de la región se vinculaban a la fluctuación económica del Norte opulento. Mientras que en el pasado una crisis financiera en el Norte provocó un colapso en el Sur, esta vez el PIB de Brasil creció 7.5 por ciento en 2010 y el de Argentina 9.2 por ciento (después del virtual colapso en 2001 del modelo neoliberal extremo). Al principio parecía que estaba ocurriendo el «desacoplamiento» de las economías latinoamericanas de la economía global, que alguna vez fue propugnado por los teóricos de la dependencia en la década de los 1960. La realidad era más prosaica pero igualmente significativa.

Como argumentan Michael Cohen y otros, en un análisis detallado del periodo, la disparidad en el desempeño durante y después de la crisis entre América Latina y el Norte es sorprendente y «resultó en recesiones relativamente más cortas y superficiales, menores aumentos en el desempleo y la pobreza, y una recuperación mucho más rápida de las tasas de crecimiento anteriores» (Cohen et al., 2012:13). En última instancia, la cuestión del crecimiento económico recae en decisiones políticas. En palabras de Karl Polanyi: «No había nada natural en el laissez-faire, los mercados libres 
nunca pudieron haber existido simplemente permitiendo que las cosas siguieran su curso» (Polanyi, 2001:145). Asimismo, la marcha atrás en la denigración neoliberal del Estado y la subordinación de la sociedad al mercado es un proceso eminentemente político.

\section{Igualdad}

En años recientes, la Comisión Económica para América Latina y el Caribe (CEPAL) ha enfatizado la igualdad en sus recomendaciones de política económica: «El valor de la igualdad, junto con el de la libertad, es la forma más humanizada de asumir las tareas de la modernidad» (CEPAL, 2010:39). Hoy, la igualdad política en América Latina está siendo socavada por niveles masivos de desigualdad socioeconómica. Así pues, la igualdad y la justicia sociales se consideran de modo correcto como los mayores desafíos en la región para el desarrollo democrático. La CEPAL plantea esta tarea en términos algo tecnocráticos —el gasto social para abordar la desigualdad debe ser visto como una inversión social en capacidades humanas-, pero es importante que sea abordada por los formuladores de políticas, incluso si se encuentra en el contexto de «crecimiento con equidad» y no por derecho propio. Esta prioridad es central en un escenario posterior a la crisis, donde se acepta un mayor grado de regulación social del mercado (como argumentaba Polanyi).

Cabe resaltar que en los últimos años ha emergido una nueva ortodoxia en los círculos creadores de políticas globales, quienes asumen que la desigualdad en América Latina se redujo de manera considerable en la década de los 1990, lo que demuestra la conveniencia del Consenso de Washington. Por tal motivo, los datos del Banco Mundial (BM) sugieren que la pobreza moderada ha decaído de 26 por ciento en 1990 a 22 por ciento en 
2004 (BM, 2007). Los datos de la CEPAL van más allá e indican que la pobreza extrema disminuyó de 23 a 15 por ciento entre 1990 y 2005 (CEPAL, 2010). En cierta forma esto no es inaudito, ya que con altos niveles de crecimiento se necesitaría un empeoramiento de la desigualdad de ingresos para que la pobreza no se reduzca. Además, estos hallazgos se basan en el dudoso planteamiento de que los índices de pobreza de México disminuyeron en más de la mitad en la década de 1990. Si bien las transferencias monetarias condicionadas (en concreto en Brasil) han tenido un impacto, los niveles de desigualdad en América Latina son extremadamente altos. A principios del siglo XXI, 10 por ciento de los hogares más ricos recibía cerca de un tercio del ingreso nacional, mientras que 40 por ciento más pobre de la población apenas percibía 10 por ciento (CEPAL, 2000:6); sólo Costa Rica y Uruguay lograron mantenerse fuera de ese escenario inhóspito y desigual.

De todas las formas de desigualdad, el aspecto más problemático y donde podría encontrarse el mayor impacto en términos de reducción de la pobreza es la desigualdad de género. Aunque los niveles de equidad de género en la educación están de cierto modo establecidos, es claro que, como sostiene la CEPAL, «la desigualdad sigue siendo un sello estructural del mercado de trabajo y la fuerza laboral femenina» (CEPAL, 2015:30). Mientras que las mujeres con grados más altos de educación han mostrado mayores grados de participación económica, las diferencias en el uso del tiempo entre hombres y mujeres revelan que «los costos de la participación de la fuerza de trabajo han sido cubiertos clara y exclusivamente por las mujeres» (CEPAL, 2010:30). Si se toma en cuenta el trabajo en el mundo — trabajo remunerado y no remunerado— se aprecia cuán desigual es la división de género del trabajo. Dada la insignificante participación de los 
hombres en el trabajo doméstico y en las labores del cuidado, es difícil esperar que las mujeres sean capaces de participar en el mercado laboral en igualdad de condiciones. A la fecha no existe un apoyo de los gobiernos de centro-izquierda para atender lo que, en efecto, es la división dominante en la sociedad junto con la clase social. Tal apoyo tendría un efecto masivo en los niveles de desigualdad de ingresos de los hogares.

\section{Sustentabilidad}

Comparado con el periodo de crecimiento orientado hacia adentro (19501980) y el periodo neoliberal (1980-2000), ahora se ha enfatizado con mayor firmeza en el ámbito político sobre la importancia de la sustentabilidad. El término desarrollo sustentable suele usarse para referirse a la sustentabilidad ambiental, pero también se puede aludir a la democracia sustentable. En la primera acepción, ha sido definido como «el desarrollo que satisface las necesidades de la generación presente sin comprometer la capacidad de las generaciones futuras para satisfacer las propias» (Brundtland, 1987:143). En ese sentido, América Latina ha entrado a un periodo de intensa vulnerabilidad debido a los efectos del cambio climático y se considera que en 2030 casi todos los países se encontrarán en categoría de «riesgo» (DARA, 2012). Es bien sabido, más no completamente comprendido, que los países andinos han sido afectados con severidad por el fenómeno de El Niño (Oscilación del Sur). Ahora el reto es revertir la destrucción ecológica y crear vías de desarrollo sustentable mientras se atienden las necesidades de subsistencia de una población en crecimiento.

Uno de los problemas más relevantes que afectan el desarrollo sustentable en América Latina es la dependencia de la exportación de sus 
recursos. Al examinar el cuadro 1 se observa hasta qué punto la exportación principal representa una considerable proporción del total.

\section{Cuadro 1}

Especialización exportadora en América Latina (2000)

\begin{tabular}{c|l|c|l|c|c|c}
\multicolumn{1}{c}{ País } & $\begin{array}{l}\text { Exportación } \\
\text { principal }\end{array}$ & \multicolumn{2}{c|}{$\%$} & \multicolumn{1}{c}{ Siguientes dos } & $\%$ & Primeros \\
Venezuela & $\begin{array}{l}\text { Petróleo } \\
\text { crudo }\end{array}$ & 58.9 & $\begin{array}{l}\text { Productos derivados } \\
\text { del petróleo, aluminio }\end{array}$ & 28.6 & 87.5 \\
\hline Ecuador & $\begin{array}{l}\text { Petróleo } \\
\text { crudo }\end{array}$ & 43.5 & Plátano, mariscos & 22.5 & 69.5 \\
Nicaragua & $\begin{array}{l}\text { Café } \\
\text { Paraguay }\end{array}$ & $\begin{array}{l}\text { Granos } \\
\text { de soya }\end{array}$ & 32.8 & $\begin{array}{l}\text { Algodón bruto, } \\
\text { vegetales }\end{array}$ & 17.8 & 50.6 \\
\hline
\end{tabular}

Fuente: adaptado de Warwick y Silva (2004:121).

Si bien las cifras agregadas para América Latina muestran que la exportación de bienes primarios se ha reducido de 90 a 42 por ciento en 2000, en cuanto a los países del cuadro 1 la mitad de sus exportaciones son bienes primarios renovables y, en el caso de petróleo crudo, no renovables. De modo semejante al énfasis anterior en el impacto negativo de la «economía de enclave», este tipo de desarrollo tampoco es sustentable porque los productos (petróleo y minerales) no son renovables; socialmente tampoco lo son porque es capital intensivo y no genera mucho empleo ni vínculos en la economía en general.

El daño ambiental por la explotación de los productos primarios en América Latina es también un gran problema. La deforestación en Brasil y 
el agotamiento de los recursos pesqueros en Chile son dos ejemplos sobresalientes. La minería en gran escala y la industria del petróleo han tenido una amplia repercusión ecológica en la contaminación del aire y los sistemas fluviales con efectos negativos en el ecosistema y la salud humana. Sin embargo, las políticas de desarrollo sustentable deben tomar en cuenta la sustentabilidad social y el contexto general de desigualdad. Murray y Silva sostienen: «La necesidad extrema obliga a la gente a abusar de los recursos naturales, en especial los renovables. Las densas poblaciones de pobres son forzadas continuamente a talar los bosques para obtener combustible y terrenos en ciclos de roza y quema» (2004:129). La aplicación de los modelos ambientales del Norte no necesariamente funciona bien en este contexto; en tanto que, por lo menos en teoría, las estrategias de «crecimiento con equidad» sí lo hacen. El crecimiento económico, la pobreza y la desigualdad y la degradación ambiental se hallan interconectados, por lo que es imprescindible el desarrollo de estrategias sustentables y progresivas que sean políticamente viables para atenderlas.

También es preciso aclarar que en América Latina se han dado grandes pasos en materia ambiental. A la par de la Conferencia de las Naciones Unidas sobre Cambio Climático 2015 en París, Uruguay anunciaba que las energías renovables proveían 95 por ciento de la electricidad del país. En menos de diez años Uruguay redujo de manera drástica su huella de carbono y disminuyó los costos de la energía. Esto se compara con una situación previa en la que casi 30 por ciento de las importaciones del país fueron contabilizadas por el petróleo. 


\section{Gobernanza}

Después del crecimiento, la igualdad y la sustentabilidad, el cuarto desafío crítico del desarrollo es la gobernabilidad. En la era de la globalización, el gobierno es más complejo de lo que solía ser: desde «afuera» poderosas fuerzas económicas restringen la toma de decisiones y desde «adentro» el Estado se encuentra «vacío» por las reformas neoliberales de los 1990. Por lo tanto, cada vez se hace referencia a la gobernanza con el propósito de implicar un proceso de «dirección» (en lugar de dictado) de la sociedad y la economía. El primer punto a destacar es que desde la redemocratización en la segunda mitad de los 1980, América Latina se ha caracterizado por una gobernabilidad democrática bastante estable, con muy pocas amenazas de intervención militar y ninguna en los países más grandes, donde el gobierno militar había durado décadas. La mayoría de las repúblicas latinoamericanas pueden denominarse «poliarquías», pues la participación es alta y el poder es distribuido entre grupos organizados y competentes, es decir, son regímenes basados en elites políticas que reconocen la necesidad de poner límites a su poder. En general, se caracterizan por elecciones libres y competitivas, así como por la libertad de expresión y de asociación que las hacen posibles.

Tras el colapso de las luchas armadas en numerosos países, a menudo traumatizantes, la izquierda comenzó a priorizar la democracia, que empezó a perder el epíteto de «burguesa» a medida que se revalorizaba la democracia liberal. Hoy, el Estado de derecho es universalmente considerado un requisito para la democracia y la buena gobernanza. Significa, ante todo, que los derechos civiles básicos sean aplicados de forma equitativa a todos los miembros de la población. Pero la larga noche de las dictaduras creó 
una cultura de impunidad entre ricos y poderosos. Ser económicamente seguro y socialmente poderoso significa estar «por encima de la ley». Hay una cultura arraigada con fuerza entre los poderosos, en la que obedecer la ley sólo es una obligación que deben seguir los pobres o los estúpidos; pagar impuestos, o de alguna otra manera ser parte del marco legal de la sociedad, con frecuencia es considerado como criminalmente descabellado para cumplirlo por voluntad propia. Somos conscientes, por supuesto, de que si bien la ley, que consagra ciertos derechos formales, puede emancipar individuos y empoderar movimientos sociales, refleja de modo inevitable las relaciones de poder más amplias en la sociedad. También es visible cómo el desarrollo democrático requiere diferentes formas de democracia además de aquellas asociadas a las tradiciones liberales del Norte.

Para la izquierda hay asuntos bajo la rúbrica de la gobernanza que no son fáciles de tratar, pero pueden evitarse, por ello una expresión común de los círculos políticos internacionales es que América Latina se enfrenta a un desafío clave con el interés de crear un mejor «clima para los negocios», atendiendo a «derechos de propiedad mal definidos, regulaciones complejas e inconsistentemente aplicadas, y sistemas judiciales impredecibles» (Ferranti y Ody, 2015:23). Es cierto que desde el punto de vista de los tribunales estadounidenses que otorgaron concesiones extraordinarias a los «fondos buitre» que impugnaban el acuerdo posterior a la crisis de Argentina de 2001, éste podría ser visto como un problema fundamental. Desde una perspectiva latinoamericana, resulta menos cierto, aunque las regulaciones claras y un poder judicial independiente son de interés para todos. El otro gran desafío mencionado a escala internacional es el tema de la seguridad ciudadana y el tráfico de drogas. Una vez más, al atestiguar la manera en que Estados Unidos ha explotado la «guerra contra las 
drogas» por ambiciones imperiales y para uso de grupos contrainsurgentes, es posible ser escépticos. Sin embargo, ningún movimiento o gobierno progresista puede hacer caso omiso de la terrible situación de la seguridad ciudadana en numerosas partes de América Latina, un problema central dentro del desarrollo democrático.

\section{Estrategias}

Frente a los desafíos ya mencionados para el desarrollo democrático en América Latina, ¿cuáles son las principales estrategias o proyectos de desarrollo emprendidos? Durante un largo periodo histórico (1950-1980, aproximadamente) prevalecía el modelo de modernización desarrollista. Su sucesor desde 1980 hasta 2000 fue el modelo neoliberal que logró una hegemonía indiscutible como el único camino hacia el desarrollo, y cuya sombra aún es proyectada sobre los acontecimientos por razones de dependencia, y porque las fuerzas sociales que se beneficiaron del modelo todavía conservan el poder (véase Munck, 2012). Algunos países como México y Colombia (y de cierta forma Chile) deambulan por ese camino y buscan una integración ventajosa aunque subordinada con Estados Unidos y el orden económico global. No obstante, desde el cambio de siglo, han surgido varios proyectos o modelos alternativos de desarrollo, ninguno es hegemónico y algunos se yuxtaponen. De ellos, sobresalen cuatro:

1. Neoextractivismo. Enfocado en exportaciones de minerales, petróleo y la agroindustria para generar ingresos.

2. Neodesarrollismo. Una repetición del modelo de desarrollo dela década de 1950 que hace concesiones al modelo neoliberal más favorable al mercado. 
3. Buen Vivir. Un nuevo modelo andino enfocado en la sustentabilidad y la recuperación de normas establecidas antes de la conquista.

4. Socialismo del siglo XXI. Una agenda nueva, o revivida, procedente principalmente de Venezuela, que articula un socialismo para la actualidad.

Cada uno de los modelos ideales será presentado y evaluado con relación a los desafíos aludidos.

\section{Neoextractivismo}

Mientras que los procesos políticos ocurridos en las últimas décadas en Venezuela, Ecuador y Bolivia han transformado el modelo de desarrollo en cierta medida, todavía dependen económicamente de la extracción de recursos naturales y de las rentas petroleras. Estos gobiernos de centro-izquierda se han caracterizado por tensiones persistentes entre estrategias neodesarrollistas —el objetivo de perseguir el crecimiento y el desarrollo en la línea del modelo anterior, aunque con un discurso más social - y una estrategia de desarrollo alternativo vista como una búsqueda de una forma de desarrollo verdaderamente humana y potenciadora. Asimismo, han intentado incrementar el crecimiento económico a través de la explotación de energía, hidrocarburos, minería y agronegocios; sin embargo, existe una tensión significativa entre la política minera dominante, por ejemplo, y los principios del Buen Vivir. El presidente de Ecuador, Rafael Correa, argumentó que el petróleo «puede ser una bendición» siempre y cuando las rentas se redistribuyan en el resto de la economía; para otros la promoción de la inversión extranjera en la minería, con todas las consecuencias humanas y ambientales que genera es una 
clara demostración de la contradicción entre el discurso radical y las prácticas desarrollistas convencionales de los nuevos gobiernos de izquierda.

El largo periodo de crecimiento económico estable bajo un Estado oligárquico (1860-1930, aproximadamente) se sustentaba en un modelo de exportación agro-minero que era económicamente dinámico y socialmente excluyente. Desde los 1990 se produjo un giro en muchas regiones de América Latina hacia un modelo neoextractivista, ya sea que impulse la exportación de gas y petróleo o soya y trigo. Está basado en la subordinación al mercado internacional, difícilmente podría ser de otra forma, y debe sujetarse a las variaciones de los precios de mercado de tales productos. En algunos casos, el Estado se involucra en dicho proceso y los gobiernos nacionales progresistas pretenden usar esas rentas en la inclusión y la redistribución social. Para los críticos de los gobiernos orientados hacia la izquierda, se trata de una nueva forma de imperialismo que se está recreando, pero sus defensores y varios analistas independientes responden que es una crítica ahistórica (véase Gudynas, 2009). Es evidente que los proyectos de desarrollo se forjan dentro de los parámetros de los patrones nacionales y geográficos ya preestablecidos y no pueden ser alterados por mera voluntad política.

Hoy en América Latina el neoextractivismo se ha convertido en un elemento integral del proyecto de desarrollo al propiciar antiguas dependencias mientras se busca un orden político más híbrido. Los crecientes ingresos generados por el petróleo o la minería — basta pensar en Venezuela y Bolivia — han permitido ambiciosos programas de redistribución social presentados como «la voluntad del pueblo». Podría sostenerse entonces que el modelo de crecimiento neoextractivista representa un retorno al Estado desarrollista, incluso defensores de los gobiernos actuales en 
Bolivia podrían argumentarlo. El Estado de desarrollo clásico promueve activamente el desarrollo (frente al Estado mínimo del neoliberalismo), media entre intereses en competencia (por ejemplo, con los ambientalistas) y proporciona legitimidad política (en contra del antiguo Estado oligárquico). Sea como fuere, una perspectiva a largo plazo apunta hacia los costos de una integración, claramente asimétrica, al mercado mundial y la contradicción fundamental con la sustentabilidad ambiental (véase Burchardt y Dietz, 2014). Con seguridad continuará siendo un proyecto de desarrollo dominante, aunque subyacente, en el corto y el mediano plazo.

Basado en el reconocimiento de los errores de la izquierda en el pasado, la búsqueda de una forma de desarrollo más allá del neoliberalismo y la incorporación de nuevas interrogantes sobre las posibilidades del crecimiento económico en una era de globalización, participación política, medio ambiente, cultura y mujeres, el nuevo socialismo latinoamericano rechaza los problemas asociados con previos modelos socialistas y propone un socialismo relevante a los tiempos contemporáneos y las particularidades de los gobiernos latinoamericanos.

\section{Neodesarrollismo}

El proyecto de desarrollo conocido como neodesarrollismo retoma el proyecto desarrollista de los 1950 al tiempo que incorpora críticas formuladas en su contra por las teorías neoliberales en la década de 1990. El viejo Estado desarrollista no regresó a plena potencia, pero reconoció que el mercado no podía por sí solo regular la economía o generar desarrollo. Los asuntos vitales de igualdad social y la necesidad de combatir la pobreza salieron de nuevo a la luz. En la era neoliberal existía una creencia 
implícita de que había ganadores y perdedores en el sistema del mercado y que estos últimos eran un daño colateral. Ante todo, el neodesarrollismo volvió a incorporar en la agenda el desarrollo nacional y regional. Para el neoliberalismo, el desarrollo nacional era un anacronismo, o peor aún, un impedimento; lo que contaba era la expansión global del mercado sin interferencias de Estados o naciones. En efecto, tal perspectiva constituye un paradigma posneoliberal, pese a que reconozca las críticas al modelo desarrollista de la década de 1950, tales como el proteccionismo excesivo y dirigismo del Estado.

El proyecto neodesarrollista aceptó los imperativos de la competitividad en la nueva economía global y también reconoció la necesidad de una reforma institucional, no sólo de la burocracia estatal. Deben tenerse en cuenta las peculiaridades de este periodo a aquel que creó la perspectiva desarrollista original, de la que se derivó la dependencia como una consecuencia radical. En los años de 1970 hubo un lapso en el que la Revolución cubana —y la URSS que la apoyaba — gozaba de una amplia credibilidad en América Latina; en contraste, los 1990 arribaron después del colapso total de la alternativa global al capitalismo, a saber, el régimen soviético y el de sus satélites. Así, el neodesarrollismo fue muy valiente al articular las políticas posneoliberales y no es de extrañar que no incorporase posiciones dependientes. No obstante, diversos autores han argumentado que el neodesarrollismo es simplemente una variante del neoliberalismo (Petras y Veltmeyer, 2001) o el «rostro humano» del neoliberalismo en el Posconsenso de Washington (Green, 1995). En cualquier caso es importante reconocer la complejidad y las raíces históricas de dicha visión.

En palabras de Gwynne y Kay, «el neoestructuralismo no debe interpretarse como la rendición de los estructuralistas al neoliberalismo, 
sino como un intento de llegar a un acuerdo con la nueva realidad de la globalización y formar la exitosa experiencia de desarrollo de los países recientemente industrializados del Este asiático» (2004:263). Una simple repetición de las políticas de desarrollo nacional de los 1950 detrás de barreras proteccionistas no sería posible en la actualidad. La relevancia de la inversión extranjera directa (IED) fue aceptada por los primeros desarrollistas, pero es más contundente en la versión actual. La trascendencia de la educación, la innovación, la reducción de pobreza, la estabilidad fiscal, la reforma del Estado, el imperio de la ley — que son centrales al neodesarrollismo-, no puede equipararse con el neoliberalismo sólo porque no son un programa socialista. Para las economías latinoamericanas tampoco se ha desvinculado una factible - y ciertamente no progresista. De las distintas opciones al neoliberalismo, el neodesarrollismo parece ofrecer la opción más viable para el crecimiento económico y la democratización política.

\section{Socialismo del siglo XxI}

El socialismo del siglo XXI fue un proyecto propuesto en principio por el presidente Hugo Chávez en Venezuela, alrededor de 2005. Fue apoyado en varias medidas por los gobiernos más radicales de izquierda, entre los cuales destacan Bolivia, Ecuador y, en menor grado, Argentina. Se basa en la necesidad de un nuevo régimen de desarrollo que ofrezca una ruptura con los regímenes neoliberales previos, y con un nuevo tipo de socialismo que planteaba un modelo nuevo y distinto de los anteriores socialismos de la URSS, China y Cuba (véase Harnecker, 2012). La búsqueda de un modelo de desarrollo alternativo surgió de la crítica y la reevaluación 
de las estrategias socialdemócratas del pasado respaldadas en el reconocimiento de los errores de la izquierda, a fin de superar el neoliberalismo e incorporar nuevas cuestiones sobre las posibilidades de crecimiento económico en una era de globalización, participación política, medio ambiente, cultura y mujeres. El nuevo socialismo latinoamericano rechazó los problemas asociados con los antiguos modelos socialistas y propuso un socialismo relevante para los tiempos contemporáneos y las particularidades de los gobiernos latinoamericanos.

Una idea clave es el nuevo enfoque de la relación entre el Estado y la sociedad civil, el cual rechaza las nociones previas de Estado y sociedad civil, como el mandato antidemocrático y la negación de los derechos civiles conectados con otros socialismos, el corporativismo del Estado desarrollista y el concepto individualista de Estado y sociedad que prevalece bajo el neoliberalismo. Se ha defendido la necesidad de desdeñar muchas formas de organización política de izquierda, por ejemplo el vanguardismo obrero de partidos y movimientos. En cambio, los nuevos gobiernos de izquierda han creado tipos de espacios políticos en alianza con movimientos políticos y sociales, que abogan por un enfoque revitalizado de democracia sustentado en «procesos constitucionales radicales» (Cicariello, 2013:128) y una configuración distinta de la relación entre Estados y movimientos sociales, «un proceso creativo constituyente de experimentación e innovación democrática» (Beasley, Cameron y Hershberg, 2012:21). En el caso de Bolivia, un aspecto central de este enfoque es, como afirma García Linera, el «proyecto de auto representación de los movimientos sociales de la sociedad plebeya» (Rockefeller, 2007:166). De acuerdo con esa idea, la participación política de las comunidades marginadas no solamente es una cuestión de reunir apoyo para los partidos políticos, ya sea que estén encabezados por 
liberales, conservadores o por una «vanguardia» proletaria; en su lugar, deben ser protagonistas de una nueva forma de poder que involucre una «relación dinámica y cambiante entre movimientos y el Estado» (Ciccariello, 2013:127). Se rehúsan a la manipulación de movimientos indígenas del pasado, al papel del partido de vanguardia y a la aplicación dogmática de la teoría con poca o nula aplicación a la realidad social latinoamericana. En contraste con el énfasis del capitalismo neoliberal sobre el individuo, el socialismo del siglo XXI posee un fuerte componente moral y ético que promueve el bienestar social, la fraternidad y la solidaridad social.

Hasta qué punto la alternativa socialista ha cumplido los desafíos planteados con anterioridad es un tópico disputado con opiniones muy polarizadas que también se expresan —o quizá especialmente- desde la izquierda. Por ahora, debe considerarse si la opción «bolivariana» planteada por Chávez es una opción política sostenible y si la naturaleza populista y personalista de muchos gobiernos de izquierda puede cumplir con los desafíos de la gobernabilidad democrática a largo plazo. Sin embargo, es importante reconocer los elementos verdaderamente novedosos que este hilo ha traído al debate político en América Latina.

\section{Buen Vivir}

Desde 2000, América Latina ha vivido una primavera política sin precedentes por la profundidad y amplitud de la transformación social progresiva. Han surgido nuevos paradigmas de cambio social y experimentación política que tal vez tengan una significación global. Lo que el enfoque de dependencia representó para la década de 1970 concerniente al paradigma de desarrollo general, es lo que hoy significa el concepto de 
Sumak Kawsay (Buen Vivir) — traducido de modo imperfecto al inglés como Good Living o Living Well. Este concepto captura muy bien el filo radical del pensamiento alternativo actual (véase Acosta, 2010; Radcliffe, 2012). En esencia habla de la reproducción ampliada de la vida más que la del capital. Defiende un modelo civilizatorio diferente al del capitalismo individualista, donde tienen prioridad los valores comunitarios y el respeto por la naturaleza. Es un paradigma de desarrollo consagrado en las constituciones de Ecuador y Bolivia, y ha causado diversos efectos en toda la región. Sumak Kawsay trabaja sobre la premisa de que hay dos transiciones en curso en América Latina: una, relativamente reciente hacia el socialismo, apenas de cien años de edad; otra, a largo plazo del colonialismo que se remonta al siglo XV. El fin de todas las manifestaciones de racismo y una mayor autodeterminación integran la lucha a largo plazo. No niega en absoluto la relevancia de las formas occidentales de democracia representativa, pero añade la necesidad de modelos de democracias participativas y comunales. Si bien la nueva cosmovisión no sólo rearticula las antiguas prácticas indígenas y se caracteriza por un hibridismo profundo, representa un desafío al eurocentrismo. Estructura nuevos principios de producción y propiedad, identidad y subjetividad y, no menos relevante, una nueva manera de entender el mundo y producir conocimiento.

La filosofía del Buen Vivir expresa un enfoque crítico de la ideología del progreso y la búsqueda de alternativas al desarrollo contemporáneo como el crecimiento económico, la explotación de la naturaleza y una sociedad basada en el materialismo y el consumismo. Supone una ruptura con ideologías y prácticas de desarrollo anteriores y ofrece «una oportunidad para construir colectivamente un nuevo régimen de desarrollo» (Acosta, 2010:39). Reconoce los valores inherentes en la naturaleza y la 
expresión de culturas y conocimientos de indígenas oprimidos o subordinados. En Ecuador, el Buen Vivir es visto como un conjunto de derechos entre los que se incluyen aquellos relacionados con salud, vivienda, educación, alimentación y medio ambiente; mientras que en Bolivia es un principio ético-cultural, a la par de la dignidad, la libertad, la solidaridad y la reciprocidad. Busca enlazar las necesidades del desarrollo con un criterio ecológico y promueve una ética que subordina los objetivos económicos a criterios ecológicos. Adicionalmente, reconoce las diferencias culturales y de género al posicionar el interculturalismo como un principio rector, y aboga por nuevas estrategias a fin de asegurar la soberanía alimentaria, el control de los recursos naturales y el agua como un derecho humano. También ha puesto en evidencia en América Latina la trascendencia de la biopolítica, en tanto que su concepción biocéntrica del socialismo representa una crítica del marxismo y de la teoría de la modernización. En efecto, el tema del análisis crítico se centra en qué medida esta visión del desarrollo democrático cumple con su promesa en la práctica en aquellos países que se inspiran en dicho enfoque y hasta qué punto se puede generalizar, por ejemplo, en el Cono Sur y México. Por supuesto, es factible pensar en el Buen Vivir en términos de una utopía, siempre una parte indispensable de los procesos de transformación social, tal como lo argumenta el ministro ecuatoriano René Ramírez (2010).

\section{Previsión}

Mirando hacia el futuro en América Latina, es imprescindible contemplar preceptos básicos político-metodológicos. Si el futuro latinoamericano es 
el objeto de estudio, se debe reconocer que la investigación social no es ingenua y que los hechos sociales no son sólo hechos. Más bien, la investigación social crea realidades sociales y los hechos sociales son construidos. Los métodos de investigación no describen simplemente el mundo social, sino que lo promulgan. En opinión de Law y Urry, «si la investigación social hace mundos, entonces, en cierta medida puede pensar acerca de los mundos que quiere ayudar a crear» (2004:391). Ello abre el potencial radical del programa de investigación prospectiva/futura que va más allá de una sencilla descripción del mundo tal como es. Mientras que las ciencias sociales trabajan más a menudo con el poder de representar las realidades sociales dominantes, pueden —y posiblemente deberían — trabajar hacia la presentación y promulgación de realidades sociales alternativas.

Un precepto metodológico básico que hay que seguir es reconocer la complejidad del mundo social actual. Contra el pensamiento lineal y reduccionista es preciso estar conscientes de los efectos secundarios y las externalidades en todas las estrategias políticas. En lugar de buscar intervenciones instrumentales para formar la realidad de acuerdo con las lecturas racionalistas de desarrollo, deben colocarse en primer plano los sistemas abiertos y la agenda social. De lo anterior se desprende que las lecturas políticas no pueden ser simplistas o maniqueas. Más bien, al seguir los avances metodológicos del enfoque de interseccionalidad (véase Lutz et al., 2011) resulta primordial la intersección de las identidades sociales y sistemas complejos de dominación y opresión. En consecuencia, la resistencia y la construcción de alternativas políticas no se sustentarán en experiencias, identidades o proyectos singulares. Siguiendo el avance conceptual de Ernesto Laclau sobre la noción de «articulación» (véase Howard, 2015) se requiere explorar la pluralidad de las demandas políticas y la forma en que 
constituyen una subjetividad social más amplia a través de la articulación equivalencial. El desarrollo de políticas alternativas no constituye una opción simplista, sino que implica una difícil —y nunca terminada - construcción de articulaciones hegemónicas. Tal proceso inevitablemente involucra esfuerzos antagónicos para estructurar contradicciones no clasistas y unificar demandas mediante un proceso de equivalencia que conduce a un sistema estable de significación.

En síntesis, se presenta una estrategia de investigación y acción colectiva orientada hacia la prospectiva según un análisis crítico de tendencias actuales y una aceptación de la radical indeterminabilidad del futuro (véase Inayatullah, 2013). El futuro no está predeterminado, no es predecible y se halla influenciado por nuestras acciones en el presente. En la era de la globalización hay una compleja red de cambios que no puede entenderse ni desde la modernización lineal y simplista ni desde las perspectivas de la dependencia. También se argumenta que el futuro es más abierto que estas dos perspectivas teleológicas, a pesar de que ideológicamente se encuentren en extremos diferentes.

Con frecuencia, la previsión es vista como una herramienta del Norte, parte integral de la revolución tecnológica de la década de 1960 (popularizada por Shell, por ejemplo) y descendiente del positivismo (predicción); recientemente se ha sometido a la influencia del posestructuralismo (véase Sardar, 2010) que destaca su complejidad y problematiza las unidades de análisis. A su vez la variante poscolonial busca aquello que falta en las imágenes actuales que se tienen del futuro. El enfoque de futuros alternativos se ha extendido con éxito en un entorno meridional, en especial en los influyentes escenarios de Mont Fleur, que desempeñaron un papel importante en la transición de Sudáfrica más allá del apartheid de la década de 1990 (véase 
Kahane, 2000). Hay una serie de herramientas empleadas en sentido prospectivo: exploración ambiental o de horizonte, planificación de escenarios, mapeo de rutas y método Delphi (véase GCPSE, 2014). En específico existen intentos de crear un enfoque cimentado en escenarios y diseñado para concebir mundos futuros que sean consistentes al interior y verificables al exterior en la investigación del desarrollo (véase Van Asselt et al., 2010).

Uno de esos enfoques en América Latina fue el estudio prospectivo de Francisco Sagasti, llevado a cabo a mediados de los 1990 (Sagasti, 2004). Este esfuerzo colectivo planteó dos ejes clave para trazar el futuro de América Latina: por una parte, si el contexto internacional era favorable o no; y por otra parte, el segundo eje tomaba en cuenta la calidad de la gobernanza. A partir de allí y de acuerdo con las tendencias actuales y el pensamiento futurista, los participantes identificaron los siguientes escenarios:

1. El vuelo del cóndor, basado en un contexto internacional positivo, buena gobernanza y avances científicos.

2. El delfín herido, sustentado en un contexto positivo internacional que no puede aprovecharse debido a la mala gobernanza.

3. El jaguar captivo, donde una economía global que mira hacia dentro limita el desarrollo pero el contexto interno es muy positivo.

4. El fénix renacido, mientras el contexto internacional es negativo, éste es superado por una estrategia de desarrollo cohesiva e inclusiva.

En un periodo de complejidad e incertidumbre tal ejercicio de escenificación puede, por lo menos, ayudar a los participantes - a través del diálogo político honesto y abierto - a establecer cuáles son los parámetros más importantes para el futuro del desarrollo y la democracia, incluso si nosotros pudiésemos estar en desacuerdo con el mejor camino a seguir. 
Desde nuestro propio análisis en las páginas anteriores, haríamos avanzar la siguiente y muy provisional matriz (abierta a la deconstrucción) para explorar los futuros alternativos de América Latina. Posicionamos dos ejes principales para «colocar» estrategias alternativas: uno horizontal centrado en un mayor o menor nivel de crecimiento económico y uno vertical basado en un mayor o menor grado de sustentabilidad. El esquema 1 matriz nos permite trazar las fortalezas y debilidades relativas de los distintos escenarios alternativos descritos anteriormente.

\section{Esquema 1}

Futuros alternativos de América Latina

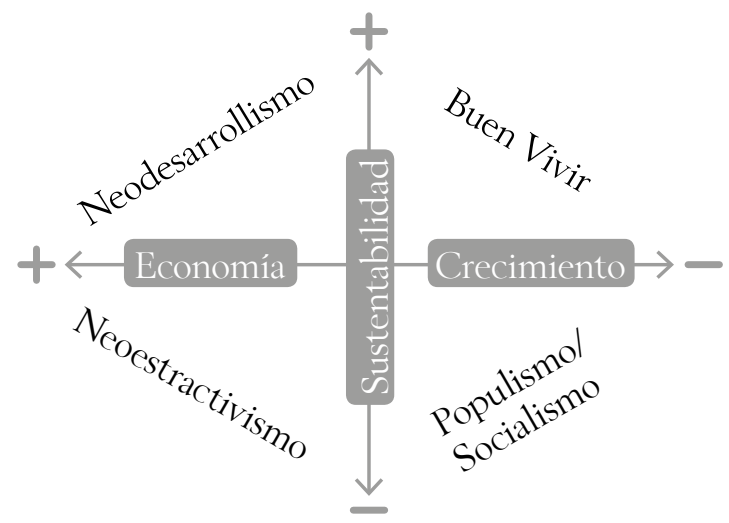

Ahora bien, estos son tipos ideales y no deben asociarse directamente con países o gobiernos actuales. El escenario del neodesarrollismo, que plantea el «crecimiento con equidad», ha hecho algunas concesiones a los modelos previos sustentados en el mercado; sin embargo, ha mostrado grados considerables de sustentabilidad, en términos políticos, lo que puede generar un fuerte crecimiento económico. Uno puede pensar en 
el Brasil de Lula da Silva e incluso en Chile bajo los socialistas (aunque en formas muy distintas) como aspirantes a ese modelo. Es la estrategia que ostenta mayores probabilidades de reclutar el apoyo de los industrialistas locales y puede generar fuertes interpelaciones nacionalistas.

Por supuesto, el neoextractivismo no se plantea como una estrategia progresiva en la transformación social, pero varios gobiernos progresistas (por ejemplo, Bolivia y Venezuela) han puesto en práctica elementos de dicho modelo. Aunque es cierto que puede producir altas tasas de crecimiento económico, es evidente que no es sustentable en términos medioambientales. Existe un debate sobre si la extracción de recursos naturales y la dependencia de la inversión extranjera configuran una estrategia de transformación social progresiva. Hay evidencia de que el boom de las materias primas ha dado lugar a un grado de redistribución social en algunos países; no obstante, una vez más, esto en realidad no es una estrategia sustentable ni en aspectos ecológicos ni económicos, puesto que los auges de las materias primas se derrumban en algún momento (por ejemplo, el precio del petróleo).

El populismo/socialismo es una amalgama de dos discursos y estrategias bastante distintos, aunque interconectados. El socialismo del siglo XXI fue el modelo promovido en Venezuela bajo el gobierno de Chávez, en tanto que el populismo puede ser mejor ejemplificado por el modo de gobierno de Kirchner en Argentina. Tal vez parecería negativo afirmar que el crecimiento económico será bajo con ese modelo, con todo y eso los patrones actuales en ambos países indican que el modelo no es económicamente fuerte. La caída del modelo de Kirchner en 2015 a manos de una inteligente campaña política a favor del mercado por parte de los partidos de oposición —incluidos los desertores de Kirchner - muestra la inestabilidad 
del modelo populista. Pese a que no es políticamente sustentable por la sencilla razón de que depende de caudillos que necesitan ser elegidos con el apoyo de la clase media, como el chavismo lo aprendió a finales de 2015.

Probablemente nos encontramos en una era posneoliberal, incluso si diversos principios neoliberales — como la apertura del comercio y la inversión al mercado global — continúan vigentes. Las políticas económicas posneoliberales (¿todavía neoliberales?) están tratando de reconectar el mercado con el ámbito social. La influencia de la crisis capitalista global de 2008-2009 y la posterior crisis europea de 2011 forma el telón de fondo de un replanteamiento de las opciones políticas latinoamericanas. Fue el impacto combinado de alrededor de 13 gobiernos de izquierda y una sociedad civil dinámica —y algunas veces rebelde - lo que alentó nuevas formas de pensamiento acerca del desarrollo en América Latina. El movimiento encaminado hacia una alternativa al neoliberalismo está más avanzado en América Latina, según Perry Anderson, porque «aquí, y sólo aquí, la resistencia al neoliberalismo y al neoimperialismo funde lo cultural con lo social y lo nacional. Es decir, implica la visión emergente de otro tipo de organización de sociedad y otro modelo de relación entre los Estados» (2004:42).

Antes de concluir es pertinente efectuar una nota reflexiva y no proclamatoria, dado que en 2015 se discernió un claro margen elevado para la «marea rosa» cuando el kirchnerismo perdió las elecciones presidenciales en Argentina; los opositores del chavismo ganaron por una «supermayoría» en las elecciones parlamentarias en Venezuela; y en Brasil, Dilma Rousseff, la sucesora electa de Lula da Silva, fue desacreditada por el parlamento. Tales acontecimientos reflejan un creciente sentimiento de desencanto con los gobiernos progresistas, semejante al que sufrió la primera 
ola de gobiernos posteriores a las dictaduras, en los 1980. Se percibía con claridad una sensación de que la corrupción estaba de vuelta, había poca fe en las capacidades de gestión económica de los gobiernos y su estilo personalista enajenó a las personas de las que recibían apoyo (por ejemplo piénsese en Chávez). Es tiempo de realizar un balance general serio de lo que se ha ganado — como la aceptación generalizada de reformas sociales y el antiimperialismo - y lo que se ha perdido, como una despolitización masiva y la reducción del socialismo a una retórica vacía (véase Gudynas, 2015), para después, quizá, empezar de nuevo con «lo que hay que hacer», acción con la que algunos analistas menos reflexivos están comenzando.

\section{Referencias}

Acosta, Alberto (2010), «El Buen Vivir en el camino del post-desarollo», Friedrich Ebert Stiftung (Policy Paper 9).

Anderson, Perry (2004), «The Role of Ideas in the Construction of Alternatives», en Borón, Atilio (ed.), New Worldwide Hegemony: Alternatives for Change and Social Movements, Buenos Aires, Consejo Latinoamericano de Ciencias Sociales.

Beasley Murray, Jon, Maxwell Cameron \& Eric Hershberg (2012), «Latin America's left turns: A tour d'Horizon», en Maxwell Cameron \& Eric Hershberg, Latin America's left turns: Politics, Policies and Trajectories of Change, Boulder, Lynne Rienner, pp. 1-22.

Brundtland, Harlem (1987), Our Common Future, Oxford, Oxford University Press. Bulmer Thomas, Victor (2014), The Economic History of Latin America Since Independence, Cambridge, Cambridge University Press. 
Burchardt, Hans Jürgen \& Kristina Dietz (2014), «(Neo-)extractivism: a New Challenge for Development Theory from Latin America», Third World Quarterly, 35(3), pp. 468-486.

Ciccariello Maher, George (2013), «Constituent Moments, Constitutional Processes. Social Movements and the New Latin American Left», in http:// www.academia.edu/3610680

Cohen, Michael, Mitchell Cook, Tanushree Dutta Isaacman (2012), «Introduction», in Michael Cohen (ed.), The Global Economic Crisis in Latin America. Impacts and Responses, London, Routledge.

Comisión Económica para América Latina y el Caribe (CEPAL) (2010), Time for Equality: Closing the Gap, Opening Trails, Santiago de Chile, Naciones Unidas. (2013), Sustainable Development in Latin America and the Caribbean: Follow up to the United Nations development Agenda. Beyond 2015 and to Rio+20, Santiago de Chile, CEPAL.

(2015), Latin America and the Caribbean: Looking ahead after the Millennium Development Goals, Santiago de Chile, CEPAL.

DARA (2012), Climate Vulnerability Monitor. A Guide to the Cold Calculus of a Hot Planet, Madrid, Fundación DARA Internacional.

De Ferranti, David \& Anthony Ody (2015), «Key Economic and Social Challenges for Latin America: Perspectives from Recent Studies», Brookings Report, in http://www.brookings.edu/wp-content/uploads/2016/06/20060803.pdf

Garretón, Antonio, Marcelo Cavarozzi, Peter Cleaves, Gary Gereffi \& Jonathan Hartlyn (2003), Latin America in the 21st Century. Toward a New Socio political Matrix, Miami, University of Miami Press.

Global Centre for Public Service Excellence (GCPSE) (2014), Foresight: The Manual, Singapore, United Nations Development Programme. 
Green, Duncan (1995), Silent Revolution: The Rise of Market Economics in Latin America, London, Cassell/LAB.

Gudynas, Eduardo (2009), «Diez tesis urgentes sobre el nuevo extractivismo: contextos y demandas bajo el progresismo sudamericano actual», en Jürgen Schuldt et al. (ed.), Extractivismo, política y sociedad. Quito, Centro de Ayuda Académica Profesional/Centro Latinoamericano de Estudios Superiores, pp. 187-225.

(2 de diciembre de 2015), «Sopesando las herencias progresistas y renovación de las izquierdas», Noticias de América Latina y el Caribe, en http:// www.nodal.am/2015/12/sepesando-las-herencias-progresistas-y-reno vacion-de-las-izquierdas-por-eduardo-gudynas/

Harnecker, Marta (2012), A World to Build: New Paths Towards Twenty First Century Socialism, New York, Monthly Review Press.

Howard, John (2015), Ernesto Laclau. Post-Marxism, populism and critique, London, Routledge.

Inayatullah, Sohail (2012), «Future Studies: Theories and Methods», in Fernando Gutiérrez Junquera (ed.), There's a Future: Visions for a Better World, Madrid, Banco Bilbao Vizcaya Argentaria, pp. 36-66.

Kahane, Adam (2000), «The Mont Fleur Scenarios What will South Africa be like in the year 2002?» Deeper News, 7(1).

Law, John \& John Urry (2004), «Enacting the social», Economy and Society, 33(3), pp. 390-410.

Lutz, Helma, María Teresa Herrera \& Linda Supik (2011), Framing Intersectionality. Debates on a Multi-Faceted Concept in Gender Studies, Surrey, Ashgate. Munck, Ronaldo (2013), Rethinking Latin America. Development, Hegemony and Social Transformation, New York, Palgrave. 
(2015), «Polanyi for Latin America: Markets, Society and Development»,

Canadian Journal of Development Studies, 36(15), pp. 1-17.

Murray, Warwick \& Eduardo Silva (2004), Latin America Transformed. Globalization and Modernity, London, Hodder Arnold.

Petras, James \& Henry Veltmeyer (2001), Globalization Unmasked: Imperialism in the 21st Century, London, Zed Books.

Polanyi, Karl (2001), The Great Transformation, Boston, Beacon Press.

Radcliffe, Sarah (2011), «Development for a Postneoliberal Era? Sumak Kawsay,

Living Well and the Limits to Decolonization in Ecuador», Geoforum (43), pp. 240-249.

Ramírez, René (2010), «Socialismo del Sumak Kawsay o biosocialismo republicano», en Los nuevos retos de América Latina: socialismo y Sumak Kawsay. Quito, Secretaría Nacional de Planificación y Desarrollo.

Rockefeller, Stuart Alexander (2007), «Dual Power in Bolivia. Movement and Government since the election of 2005», Urban Anthropology, 36(3), pp. 161-193.

Sagasti, Fernando (2004), «Thinking about the Future: Trends and Scenarios in Latin America», Development, 47(4), pp. 15-25.

Sardar, Zia (2010), «The Namesake: Futures, future studies, futurology, futuristic, foresight: what's in a name?», Futures (42), pp. 177-184.

Van Asselt, Marjolein B.A. et al. (2010), Foresight in Action Developing Policy-Oriented Scenarios, London, Earthscan. 